\title{
〈Chemische Biologie〉
}

\section{Das Chemical Genomics Centre}

\author{
„Aufklärung biologischer Prozesse mit wirkstoffartigen Molekülen als Sonden“ lautet \\ kurz gefasst das Forschungsprogramm des Chemical Genomics Centre in Dortmund.
}

Entstanden aus einer Initiative der Max-Planck-Gesellschaft und Unternehmen der Wirk-

stoffindustrie, kooperieren hier Forschergruppen aus Max-Planck-Instituten mit Expertise

in Chemie und Biologie und neu eingerichteten Nachwuchsgruppen.

\begin{abstract}
$\checkmark$ Biologische Phänomene können heute zunehmend auf molekularer Ebene untersucht werden. Viele Fragen aus der Genomforschung beispielsweise können nur in interdisziplinärer Kooperation an der Grenzfläche von Chemie und Biologie aufgegriffen und bearbeitet werden. Dabei rückt ein Forschungsansatz in den Mittelpunkt des Interesses, der zellbiologische Untersuchungen mit wirkstoffartigen Molekülen als modulierende Liganden ermöglicht. So hat der Naturstoff Brefeldin das Studium von Transportvorgängen in Zellen ermöglicht; das Alkaloid Colchicin die Modulierung der Zellteilung. Diese und andere prominente Beispiele für Molekülsonden hatten einen großen Einfluss auf ganze Arbeitsgebiete der Biologie.
\end{abstract}

Die chemischen Genomik studiert nun Genprodukte mit chemischen und biologischen Methoden. Das Auffinden von Molekülsonden ist dabei ein besonderer Schwerpunkt. Mit der Synthese von Verbindungsbibliotheken und biologischen Assays werden heute Wirkstoffmoleküle gesucht, die als solche Molekülsonden dienen können.

Die chemische Genomik erfordert, dass chemische und biologische Expertise zusammengeführt und gebündelt werden. Auch wenn Teilaspekte dieses Forschungsgebiets bereits in kleineren Kooperationen bearbeitet werden, fehlte in Deutschland und Europa doch lange ein gezieltes Programm zur Untersu- chung biologischer Phänomene mit modulierenden Liganden. Die MaxPlanck-Gesellschaft hat diese Problematik aufgegriffen und unter der Federführung von Herbert Waldmann das Chemical Genomics Centre gegründet (Abbildung 1).

\section{Das Chemical Genomics Centre}

- Mehrere Max-Planck-Forschungsgruppen aus der Chemie und Biologie arbeiten im Chemical Genomics Centre (CGC) eng zusammen, unter anderem die Gruppen von Herbert Waldmann, Roger Goody und Alfred Wittinghofer (alle MPI für moleku- lare Physiologie, Dortmund), und die Gruppe von Alois Fürstner (MPI für Kohlenforschung, Mülheim/ Ruhr). Weiterhin sind das MPI für Züchtungsforschung, Köln (George Coupland, Paul Schulze-Lefert), das MPI für molekulare Zellbiologie und Genetik, Dresden (Marino Zerial), das MPI für Psychiatrie, München (Felix Hausch), und das MPI für Biochemie, Martinsried (Thomas Mayer) beteiligt. Wichtig ist der interdisziplinäre Ansatz der Zusammenarbeit; die Expertise der jeweiligen Arbeitsgruppe wird genutzt, um ein gemeinsames Ziel zusammen zu erreichen. Ein Beispiel für Forschung in-
Abb. 1.

Im Chemical

Genomics Centre

arbeiten Max-

Planck-Institute mit

Unternehmen und

unabhängigen

Nachwuchsfor-

schungsgruppen

eng zusammen.
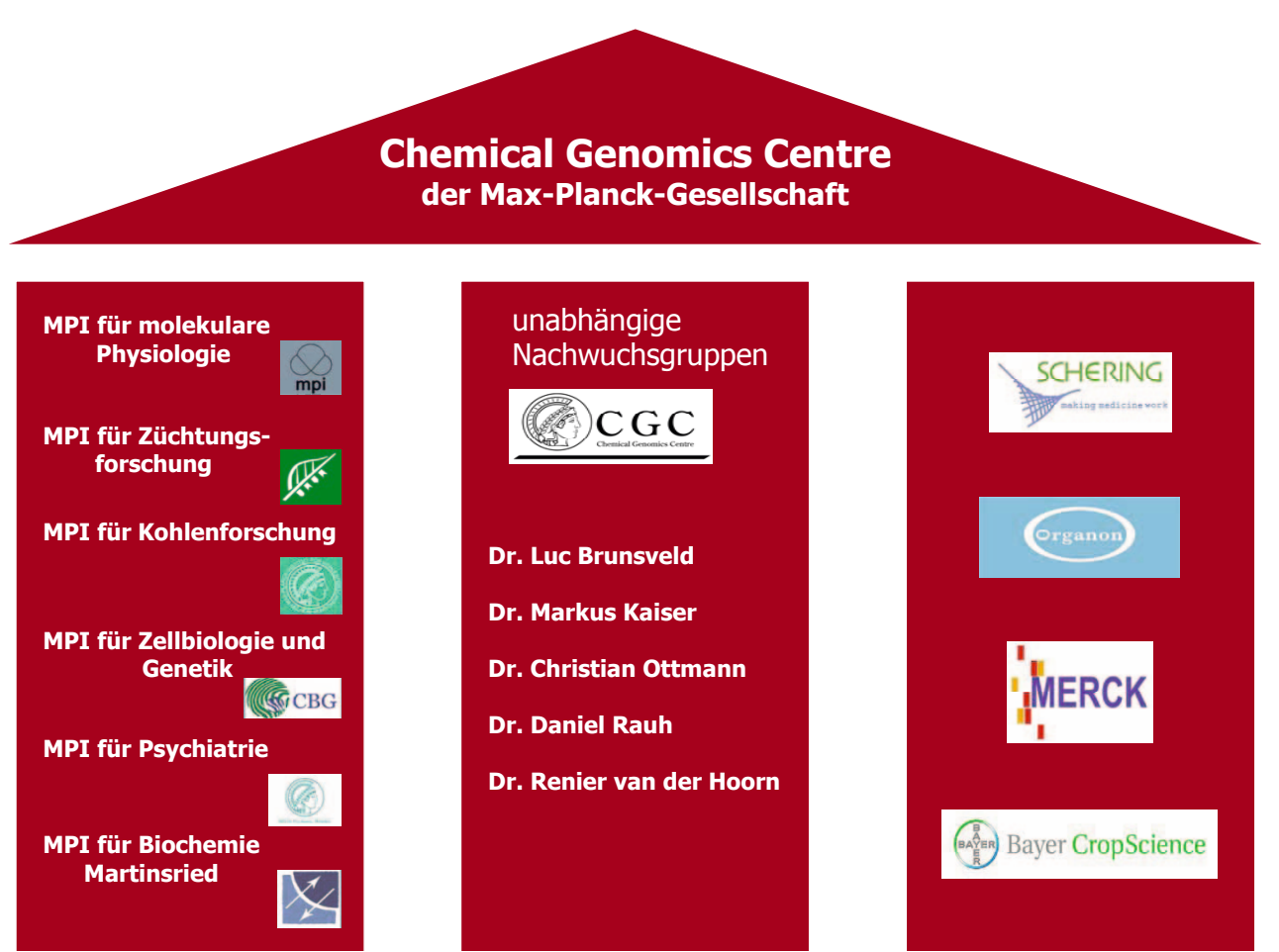

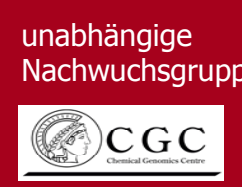

Dr. Luc Brunsveld

Dr. Markus Kaiser

Dr. Christian Ottmann

Dr. Daniel Rauh

Dr. Renier van der Hoorn

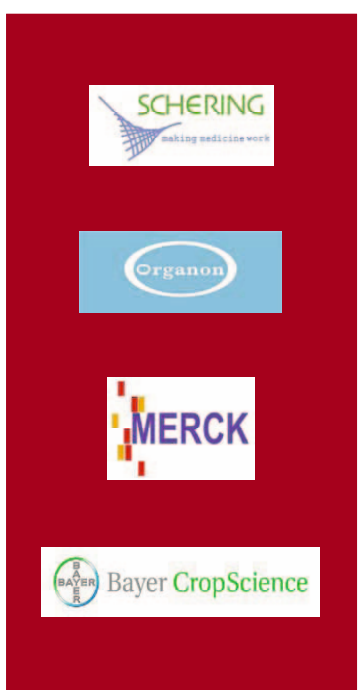


nerhalb des Chemical Genomics Centre zeigt Abbildung 2.

Darüber hinaus sind auch die Firmen Merck (Darmstadt), Organon, Schering und BayerCropScience am Chemical Genomics Centre beteiligt. Diese enge Zusammenarbeit der Max-Planck-Gesellschaft mit forschenden Unternehmen in den Life Sciences eröffnet einerseits Forschungsmöglichkeiten, die ein akademisches Umfeld normalerweise nicht bietet. Andererseits können die Firmen auf diese Weise an Grundlagenforschung in Gebieten teilhaben, bei denen noch Innovationsdefizite bestehen, die aber für spätere Anwendungen hochrelevant sind. Die Beteiligung der Unternehmen sichert auch die Fokussierung des Chemical Genomics Centre auf den Forschungsfeldern Gesundheit, Pflanzenschutz und Ernährung.

\section{Eine Plattform für Talente}

$\checkmark$ Nachwuchsförderung ist ein weiteres wichtiges Anliegen des Chemical Genomics Centre. Die MaxPlanck-Gesellschaft und die beteiligten Unternehmen haben zusammen unabhängige Nachwuchsgruppen eingerichtet, in denen junge Chemiker und Biologen fünf Jahre gefördert werden sowie eigenständige und unabhängige Forschung betreiben (Abbildung 3). Im folgenden werden die Arbeitsgebiete der Nachwuchsgruppen vorgestellt.

Luc Brunsveld und seine Mitarbeiter beschäftigen sich mit der chemischen Biologie von Protein-Protein-Wechselwirkungen. Im Fokus steht dabei die Wechselwirkung zwischen Kernrezeptoren (im Zellkern lokalisiert) und ihren Cofaktoren Screeningverfahren für diese Wech-
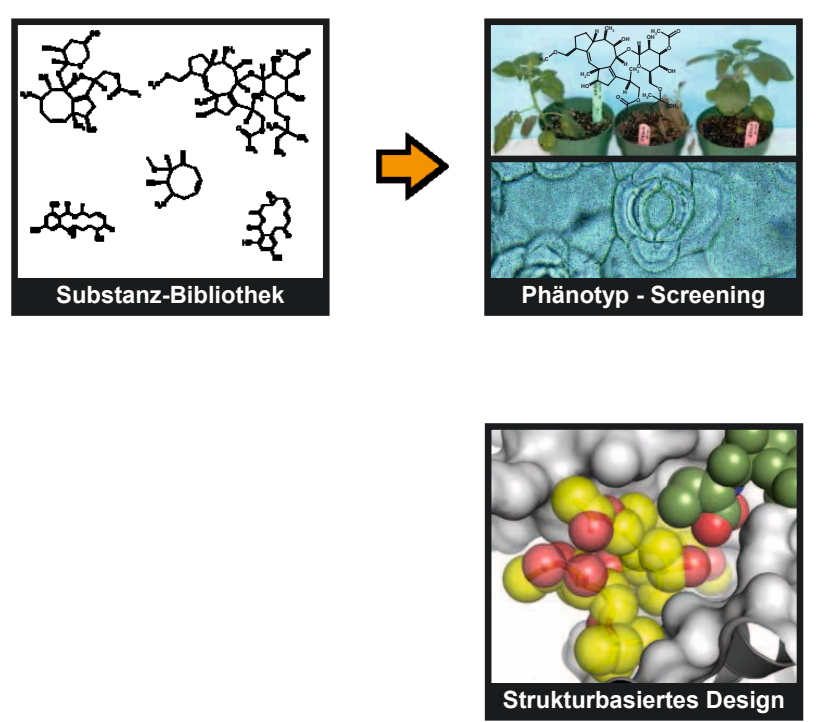
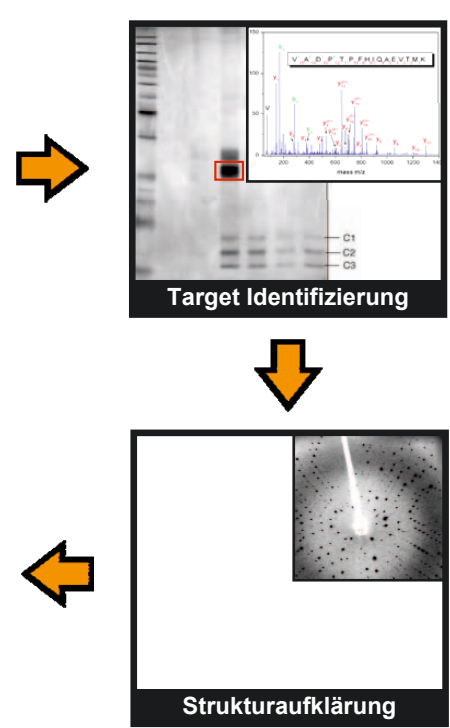

Abb. 2. Chemische Genomik am Beispiel des Protein-Protein-Wechselwirkungs-Stabilisators Fusicoccin. 1. Substanzbibliothek: Ausgehend von einer Reihe chemisch diverser Strukturen wird deren Effekt auf das Pflanzenwachstum untersucht. 2. Phänotypischer Screen: Einige der getesteten Substanzen führen zu einem auffälligen Phänotyp. Wie bei der mittleren Pflanze zu sehen, kommt es zu einem Vertrocknen der Blätter. Die morphologische Untersuchung der Pflanze weist die permanente und maximale Öffnung der Stomata (Spaltöffnungen) als Grund für den massiven Wasserverlust aus, der den Vertrocknungszustand der Pflanze hervorruft. Stomata sind essentielle Pflanzenorgane, die dem Gasaustausch dienen. Hierbei muss die Pflanze einen Kompromiss zwischen der für die Photosynthese notwendigen $\mathrm{CO}_{2}$-Aufnahme und der Gefahr des übermäßigen Wasserverlustes durch Transpiration eingehen. 3. Targetidentifizierung: Mit zweidimensionaler Gelelektrophorese und massenspektrometrischer Analyse wurde als zelluläres Target der Welke-induzierenden Verbindungen die Protonen-ATPase der pflanzlichen Plasmamembran identifiziert. 4. Strukturbiologie: Die Strukturbiologie liefert ein Verständnis der atomaren Wechselwirkung zwischen dem aktivem Agens und dem identifizierten Zielprotein. 5. Strukturbasiertes Design: Anhand der Kristallstrukturanalyse konnte die wirkaktive Substanz als ein Stabilisator von Protein-Protein-Wechselwirkungen identifiziert werden. Der gefundene sehr komplexe Naturstoff (durchsichtiges Kalottenmodel) stabilisiert die Protein-Protein-Wechselwirkung bestehend aus einem Adapterprotein (weiße Oberfläche) und der ProtonenATPase (grüne Kalotten). Die Komplexstruktur aus Ligand und Protein dient als Startpunkt für das rationale Design neuer kleiner Moleküle mit optimierten Eigenschaften wie Größe, Affinität und Selektivität.

selwirkung sind aufgebaut worden, z. B. Fluoreszenzpolarisation und OnBead-Screening. Sie dienen dazu, kleine Modulatoren zu finden, um die biologische Funktion dieser wichtigen Klasse von Proteinen weiter zu erforschen. Mit der Pharmaindustrie bestehen hier Kooperationen z. B. bei der Proteinkristallographie und der computerunterstützten Chemie.

In der Arbeitsgruppe von Markus Kaiser werden Wirkstoffsonden synthetisiert, die auf Naturstoffen mit bekannten Wirkprinzipien basieren. Ziel der Synthesen ist die Entwicklung von Molekülen zur Modulation von Enzymaktivitäten, insbesondere zur chemischen Regulation von Proteolysesystemen der Proteinqualitätskontrolle und der pflanzlichen Immunabwehr. Evaluiert werden die Modulatoren sowohl durch In-vitro als auch In-vivo-Screens. Externe Kooperationen mit strukturbiologischen Arbeitsgruppen helfen, die Wechselwirkungen zwischen der Wirkstoffsonde und den Zielproteinen aufzuklären und damit die Modulatoren weiter zu optimieren.

Die Gruppe von Christian Ottmann beschäftigt sich mit Stabilisatoren für Protein-Protein-Wechselwirkungen. 14-3-3 Proteine z.B. sind Adapterproteine, die an der Regulation von mehreren hundert Zielproteinen durch direkte Bindung beteiligt sind, u. a. der Raf Kinase, der Zellzyklus-Phosphatase Cdc25, des proapoptotischen Regulatorproteins BAD und von FOXO-Transkriptionsfaktoren. Die Stabilisierung solcher Proteinkomplexe ist zum einen für die Untersuchung von Signaltransduktions-Netzwerken von Bedeutung, zum anderen lassen sich therapeutisch nutzbare Effekte mit solchen Molekülen erreichen.

Die Arbeitsgruppe von Daniel Rauh beschäftigt sich mit der Entwicklung und Anwendung neuer Techniken zur Verfolgung der Funktionsweise von Proteinen mit chemischen Sonden in lebenden Zellen. Im Mittelpunkt stehen dabei die zeit- und raumaufgelöste Beschreibung von Aktivitätsverteilungen von Protein-Kinasen und Reduktasen innerhalb von Signaltransduktionskas- 
